\title{
An investigation into the avoidability of drug reaction with eosinophilia and systemic symptoms (DRESS) syndrome
}

Mohammed Danjuma ( $\sim$ mdanjuma21@gmail.com )

Weill Cornell Medicine

Lina Naseralallah

Hamad Medical Corporation

Bodoor AbouJabal

Hamad Medical Corporation

Mouhand Mohamed

Hamad Medical Corporation

Ibrahim Abubeker

Brown University

Layla Jabbar

Hamad Medical Corporation

Abdelnaser Elzouki

Qatar University

\section{Research Article}

Keywords: avoidability, DRESS syndrome, LAAT, RegiSCAR, ADR

Posted Date: April 16th, 2021

DOl: https://doi.org/10.21203/rs.3.rs-408287/v1

License: (9) (i) This work is licensed under a Creative Commons Attribution 4.0 International License.

Read Full License 


\section{Abstract}

Purpose Drug reaction with eosinophilia and systemic symptoms (DRESS) syndrome is a rising morbidity amongst hospitalized patients. Significant uncertainty remains regarding its exact diagnostic tool. Whilst algorithms for management of index cases have been well established, examination of its potential preventability by utilizing novel "avoidability" tools have remained unexplored.

Methods This prospective study is comprised of patients who presented to emergency department of Weill Cornell Medicine-affiliated Hamad General Hospital, Doha Qatar with suspected DRESS syndrome. These cases were comparatively adjudicated and ascertained independently using RegiSCAR, and JSCAR tools by two clinical pharmacists and two General Physicians. They were subsequently rated for potential avoidability utilizing the Liverpool adverse drug reactions avoidability tool (LAAT) by the same team of raters.

Results A total of 16 patients satisfy RegiSCAR criteria for DRESS syndrome. The mean age of the study population was 41.5 (SD \pm 13.3 ) years, with a disproportionately male phenotype $(n=12 ;[75 \%])$. The median latent period from drug ingestion to clinical presentation was 14 days (IQR 6.5, 29). The median RegiSCAR and J-SCAR scores were 6 (IQR 5, 6.8), 5 (IQR 4, 5.8) respectively. Utilizing the LAAT tool, about $60 \%$ of the DRESS syndrome-drug pairs were rated as "avoidable" ("probable" or "definite"). The overall Krippendorf's alpha with the LAAT was 0.81 (SE 0.10, Cl 0.59-1.00); with an intraclass correlation coefficient (ICC) of 0.90 (CI 0.77, 0.96.).

Conclusion In a randomly selected cohort of DRESS syndrome-drug pairs, the LAAT tool showed a significant proportion that were potentially avoidable ("possibly" and "definitely"). This will need validation by large sample sized prospective studies utilizing the updated LAAT tool proposed by this study.

\section{Introduction}

First recognized in 1937, drug rash with eosinophilia and systemic symptoms (DRESS) syndrome is an idiosyncratic reaction to a range of drugs; often leading to systemic manifestations including rash, fever, lymphadenopathy, eosinophilia, renal/hepatic injury (1). The temporal profile of its presentation is variable, but is suggested to range between two to eight weeks (1). The reported prevalence of clinically significant DRESS syndrome has been estimated to range between 1 in 1000, and 1 in 10,000 exposures $(1-4)$, with no discernible gender bias (5). Amongst the range of drugs thus far identified to cause DRESS syndrome, includes allopurinol (32\%), phenytoin (18\%), dapsone (17\%), vancomycin (39\%), penicillin $(13 \%)$ amongst others $(3,6)$. Multiple clinical adjudication tools and algorithms have been developed and subsequently validated to aid clinicians in the determination of the likelihood of DRESS in patients who present with clinical features that raise suspicion. These tools include the European Registry of Severe Cutaneous Adverse Reactions (RegiSCAR) (1), and the Japanese Research Committee on Severe Cutaneous Adverse Reaction (J-SCAR) (2); both of which have been exhaustively validated and are 
currently clinical use. DRESS syndrome confers significant morbidity and sometimes unacceptable excess mortality, with rates of between $5-10 \%$ reported in some series $(1,7)$. Despite these clinical burden, current attempts have focused primarily on management of each index case with paucity of work on its potential preventability. The most recent Spanish Guidelines for diagnosis, management, treatment, and prevention of DRESS Syndrome made recommendations centered around added predictive values of specific pharmacogenetic makers (such as single nucleotide polymorphisms) encoding implicated drugs (8). Recently, there has been a rising interest in the determination of the avoidability of adverse drug reactions (ADR) in the general population using novel "avoidability" determination algorithms/tools (912). One of such tools is the Liverpool adverse drug reaction avoidability tool (LAAT) (9). Despite extensive utility of this tool in the determination of ADR in other drug-related clinical risks, its potential impact on adjudication of DRESS syndrome-drug pairs have remained unexamined. The potential utility of avoidability determination in the prevention of morbidities related to ADR (such as DRESS syndrome) are enormous; including positive impact on and supporting clinical decision framework around therapeutic commissioning of specific drugs associated with increasing DRESS association and demonstrable preventability. In this study, we have for the first time explored the potential utilization of the LAAT tool in the setting of DRESS syndrome to ascertain its utility in determining the avoidability of this syndrome. The outcome of our exploratory work could further assist in the development of clinical guidelines and policies that are more proactive and focused on prevention rather than the current reactive paradigm focused primarily on management of individual patients

\section{Methods}

This prospective study was comprised of patients presenting with suspected DRESS syndrome to the emergency department (ED) of Weill Cornell Medicine-affiliated Hamad General Hospital (HMC), Doha Qatar as part of an observational adverse drug reactions cohort. From this population, a random sample of 16 cases initially adjudicated (by their primary physicians and dermatologists) for potential diagnosis of DRESS syndrome were selected as part of this current study's cohort. Socio-demographic, clinical, and laboratory parameters of the study participants were abstracted from an online patient record system (Cerner) to a Microsoft excel data collection database. Variables of interest includes age, gender, ethnicity, comorbidities, implicated drugs, drug latent periods, as well laboratory parameters required for completing the RegiSCAR and JSCAR algorithms. Implicated drugs were identified by raters based on the likelihood of causing DRESS syndrome as reported in the literature considering the time factor in the patient's hospital course. Two independent assessors determined the most likely offending agent by reviewing the patient's chart and any discrepancies were resolved by discussion, revision of the time frame, and dermatology opinion

Two independent rating pairs (2 Clinical Pharmacists and 2 General Physicians) initially assessed the likelihood of DRESS syndrome diagnosis utilizing DRESS syndrome scoring tools (RegiSCAR and JSCAR) (1). Cases with a score of $>5$ were included in the study. Subsequently, the LAAT tool was utilized by the same rating pairs to ascertain the potential avoidability of these DRESS syndrome-drug pairs (Fig. 1). The ordinal outcomes for the RegiSCAR tool were reported as "no case", "possible case", 
"probable case" and "definite case (1); while that for the JSCAR tool were reported as "consider other diagnosis", "atypical DRESS", and "typical DRESS" (2). Avoidability of DRESS syndrome outcomes were reported as "definitely avoidable", "possibly avoidable", "not avoidable" and "unassessable". The study protocol and all relevant study documentation were reviewed and approved by Hamad Medical Corp (HMC) institutional review board. All patients provided informed consent to participate in the study. All methods were performed in accordance with all relevant guidelines and regulations.

\section{Statistical analysis}

Ordinal outcomes from diagnostic, and avoidability tools are represented as numbers (\%), with their pairwise interrater agreement proportions, Krippendorf's kappa statistics with $95 \%$ confidence intervals (Cl), and intraclass correlation coefficients (ICC). To determine agreement across multiple assessors we calculated and compared the pairwise scores with a global kappa score. All statistical analyses were conducted with Stata, (StataCorp. 2019. Stata Statistical Software: Release 16. College Station, TX: StataCorp LLC).

\section{Case definition and ascertainment}

\section{DRESS syndrome}

- Patients are adjudged as having DRESS syndrome if RegiSCAR score $\geq 5$

- Extreme agreement (EA) between rating pairs was defined as a situation where both raters scored a DRESS syndrome-drug pair to the same ordinal outcome

- Extreme disagreement (ED) between rating pairs was defined as a situation where a rater adjudicates a DRESS syndrome-drug pair outcome as unassessable" whilst the other rater assessed it as any of the three other outcomes ("not avoidable", "possibly avoidable", or "definitely avoidable") (10).

- Kappa values of $\leq 0.20,0.21-0.40,0.41-0.60,0.61-0.80$, and $0.81-1$ represented a slight, fair, moderate, substantial, and almost perfect agreement, respectively (13).

\section{Results}

The baseline characteristics of the study population $(n=16)$ are shown in Table 1. A total of 16 patients satisfy RegiSCAR criteria for DRESS syndrome. The mean age of the study population was 41.5 (SD \pm 13.3) years and was comprised of disproportionately male subjects $(n=12 ;[75 \%])$. The median RegiSCAR and J-SCAR scores were 6 (IQR 5, 6.8), 5 (IQR 4, 5.8) respectively. The comparative interrater agreement (IRR) proportion between the four different raters (two General physicians and two clinical pharmacists) utilizing the RegiSCAR and J-SCAR tools were 0.4984 (Cl 0.18-0.82), 0.31 (Cl-0.07 to 0.71) respectively. The median latent period from drug ingestion to clinical presentation was 14 days (IQR 6.5, 29). Other estimates of key laboratory determinants including serum eosinophils are given in Table 1. 
Table 1

Baseline characteristics of the study population $(N=16)$

\begin{tabular}{|c|c|c|c|}
\hline Age (years) & $41.5(13.3)$ & - & - \\
\hline \multicolumn{4}{|l|}{ Mean (SD) } \\
\hline Gender (Male) & $12(75)$ & - & - \\
\hline \multicolumn{4}{|l|}{$\mathrm{N}(\%)$} \\
\hline AST Median (IQR) & 50 (27 124) & - & - \\
\hline ALT Median (IQR) & $56(22$ 160) & - & - \\
\hline ALP & $115(97,145)$ & - & - \\
\hline Albumin & $34(25,38)$ & - & - \\
\hline PT & & - & - \\
\hline Neutrophils & $2.5(23.5,67)$ & - & - \\
\hline Eosinophils $\left(\times 10^{3}\right.$ cells $\left./ \mu \mathrm{L}\right)$ & $4.5(2.4,7.5)$ & - & - \\
\hline \multicolumn{4}{|l|}{ Median (IQR) } \\
\hline $\mathrm{EGFR}^{\star 7}\left(\mathrm{mls} / \mathrm{min} / 1.73 \mathrm{~m}^{2}\right.$ & $60(60,107)$ & - & \\
\hline \multicolumn{4}{|l|}{ Median (IQR) } \\
\hline Drug latent period (days) & $14(6.4,29.2)$ & - & - \\
\hline \multicolumn{4}{|l|}{ Median (IQR) } \\
\hline Extent of skin rash $(>50 \%)$ & $9 / 16$ & & \\
\hline Internal organ involvement & 25 & & \\
\hline Liver (\%) & 37.5 & & \\
\hline \multicolumn{4}{|l|}{ Kidneys (\%) } \\
\hline RegiSCAR & $6(5,6,8)$ & & \\
\hline \multicolumn{4}{|l|}{ Median (IQR) } \\
\hline J-SCAR & $5(4,5.8)$ & & \\
\hline Median (IQR) & & & \\
\hline
\end{tabular}

\section{\#: Determined by LAAT}

SD: Standard Deviation; AST: Aspartate Aminotransferase; ALT: Alanine Aminotransferase; ALP: Alkaline Phosphatase; PT: Prothrombin time; IQR: Interquartile range; EGFR: Estimated Glomerular Filtration Rate 
Ibuprofen

Naproxen

1

Ceftriaxone

3

Piperacillin/tazobactam

Desloratadine

1

Dasatanib

1

\#: Determined by LAAT

SD: Standard Deviation; AST: Aspartate Aminotransferase; ALT: Alanine Aminotransferase; ALP: Alkaline Phosphatase; PT: Prothrombin time; IQR: Interquartile range; EGFR: Estimated Glomerular Filtration Rate

\section{DRESS syndrome Avoidability Outcomes}

The LAAT tool utilization resulted in a total of 60 outcome decisions. Of these, $6.7 \%$ were rated as "unassessable", $33.3 \%$ were rated as "not avoidable". Significantly, $60 \%$ of the decisions were rated as "avoidable" ("probable" or "definite"). The overall Krippendorf's alpha with the LAAT was 0.81 (SE 0.10, Cl 0.59-1.00). The intraclass correlation coefficient (ICC) utilizing the LAAT tool was $0.90(\mathrm{Cl} 0.77,0.96)$. The proportion of exact agreement between raters was $86.7 \%$. (95\% Cl $0.71-1.00)$. The drugs most associated with DRESS syndrome were beta lactam antibiotics $43.7 \%(n=7)$, Allopurinol $25 \%(n=4)$, and NSAIDS $18.7 \%(n=3)$.

\section{Discussion}

To our knowledge, this study represents the first examination of the potential preventability of DRESS syndrome-drug pairs by utilizing the LAAT. We found about $60 \%$ of DRESS syndrome-drug pairs were potentially avoidable ("probable" or "definite"). The exact agreement proportions between the raters in terms of avoidability was about $80 \%$; which will indicate excellent agreement. Our report is consistent with the agreement proportions reported by the original developers of the tool (9), as well subsequent validation studies $(10,14,15)$. The proportionately higher interrater agreement and avoidability proportions ( 0.80$)$ reported from our study compared to that by both Louise et al (9) and Danjuma et al (10) may be due to the phenotype of the clinical risks the tool (LAAT) was attempting to adjudicate. The fact that DRESS syndrome has a distinct cutaneous clinical phenotype may have improved the degree of certainty in both the interrater agreement as well as avoidability determination outcomes compared to that seen with other ADR's (such as drug induced liver injury (15). We do concede that some forms of DRESS syndrome are idiosyncratic in manifestation and may not be amenable to adjuication of potenail 
avoidability. However what proportion of this is attributable the latter category in our current sample population is difficult to ascertain (largely because of our small sample size).

Our initial use of both RegiSCAR and J-SCAR tools was to allow a more robust determination of the diagnosis of the likelihood of DRESS syndrome in the study subjects. This is to ensure a more rigorous adjudication of potential avoidability afterwards. Although an exhaustive comparative analyses of the determinative values of the RegiSCAR and JSCAR tools is not within the remit of this study, but we found it interesting that the interrater agreement proportions were lower with the JSCAR $(0.31)$ compared to the Regi-SCAR tools (0.50). This represents "slight" agreement between the raters with the JSCAR versus "good" agreement in the RegiSCAR tool (13). The limitations of the J-SCAR as a DRESS syndrome adjudication tool have exhaustively been discussed elsewhere including the need for demonstration of Human Herpes 6 reactivation (HHV-6) (16).

The key novelty and strength of our study lies is its seminal attempt at examination of potential avoidability of DRESS syndrome by utilizing the LAAT. Additionally, the initial determination of likelihood of diagnosis of DRESS with two of the most widely validated tools confers added rigor to the adjudication process. We deliberately made a comparison between clinical pharmacist and the General physicians, as they represent distinct specialties that are comparatively more likely to encounter this morbidity in their daily practice. Despite the relatively high agreements proportions between the rating pairs, we have identified a number of factors which may have contributed to disagreements in the adjudication of potential avoidability of DRESS syndrome-drug pairs. Some of these includes; differences (amongst raters) in the background pharmacological understanding of the implicated drugs vis-à-vis the various reported phenotypes of their adverse events profile; varying interpretation of the path of the LAAT that was open to subjective interpretation (such as questions $2 a$ and 4 in Fig. 1) amongst others.

Our study was limited by its small sample size (given its rarity) as well as the same constraints associated with the use of such data schemes; these includes the generic design of the LAAT tool, which on one hand allows clinicians to determine the avoidability of a wide range of adverse events, but opens up others such as skin dermatosis to wide range of interpretations which potentially could confound our results. Indeed, it is consequent upon this that, we propose modification of the LAAT tool to incorporate the RegiSCAR score into its avoidability determination path for subsequent studies in this area (Fig. 2). The proportion of potentially avoidable DRESS syndrome outcomes from our report if validated by larger sample sized systematic diagnostic studies will have implication for review of therapeutic commissioning and or practices around the implicated drugs.

\section{Conclusion}

In a randomly selected sample of DRESS syndrome-drug pairs, the LAAT tool showed a significant proportion that were potentially avoidable ("possibly" and "definitely"). This will need validation by large sample sized studies utilizing the updated LAAT tool proposed by this study. 


\section{Declarations}

\section{Conflict of interest}

None of the authors have any conflict of interest to declare

\section{Funding}

The study received no structural funding support

\section{Author contribution}

MID was involved in study concept development, regulatory approval, data analyses, and manuscript writing; L.M.A.N and B.A collected and analyzed data; All authors (M.I.D, L.M.A.N, B.A, L.A, I.Y.A, M.F.M, and A.E) were involved in writing and reviewing the manuscript.

\section{Availability of data and material}

All data relating to this work is available from the corresponding author on reasonable request.

\section{References}

1. Kardaun SH, Sekula P, Valeyrie-Allanore L, Liss Y, Chu CY, Creamer D, et al. Drug reaction with eosinophilia and systemic symptoms (DRESS): an original multisystem adverse drug reaction. Results from the prospective RegiSCAR study. Br J Dermatol. 2013 Nov;169(5):1071-80.

2. Shiohara T, lijima M, Ikezawa Z, Hashimoto K. The diagnosis of a DRESS syndrome has been sufficiently established on the basis of typical clinical features and viral reactivations. Vol. 156, The British journal of dermatology. England; 2007. p. 1083-4.

3. Wolfson AR, Zhou L, Li Y, Phadke NA, Chow OA, Blumenthal KG. Drug Reaction with Eosinophilia and Systemic Symptoms (DRESS) Syndrome Identified in the Electronic Health Record Allergy Module. J allergy Clin Immunol Pract [Internet]. 2018/08/31. 2019 Feb;7(2):633-40. Available from: https://pubmed.ncbi.nlm.nih.gov/30176295

4. Ramírez E, Medrano-Casique N, Tong HY, Bellón T, Cabañas R, Fiandor A, et al. Eosinophilic drug reactions detected by a prospective pharmacovigilance programme in a tertiary hospital. $\mathrm{Br} \mathrm{J}$ Clin Pharmacol. 2017 Feb;83(2):400-15.

5. Cacoub P, Musette P, Descamps V, Meyer O, Speirs C, Finzi L, et al. The DRESS syndrome: a literature review. Am J Med. 2011 Jul;124(7):588-97.

6. Sharifzadeh S, Mohammadpour AH, Tavanaee A, Elyasi S. Antibacterial antibiotic-induced drug reaction with eosinophilia and systemic symptoms (DRESS) syndrome: a literature review. Eur J Clin Pharmacol [Internet]. 2020; Available from: https://doi.org/10.1007/s00228-020-03005-9

7. Hiransuthikul A, Rattananupong T, Klaewsongkram J, Rerknimitr P, Pongprutthipan M, Ruxrungtham K. Drug-induced hypersensitivity syndrome/drug reaction with eosinophilia and systemic symptoms 
(DIHS/DRESS): 11 years retrospective study in Thailand. Allergol Int. 2016 Oct;65(4):432-8.

8. Cabañas R, Ramírez E, Sendagorta E, Alamar R, Barranco R, Blanca-López N, et al. Spanish Guidelines for Diagnosis, Management, Treatment, and Prevention of DRESS Syndrome. J Investig Allergol Clin Immunol. 2020;30(4):229-53.

9. Bracken LE, Nunn AJ, Kirkham JJ, Peak M, Arnott J, Smyth RL, et al. Development of the Liverpool Adverse Drug Reaction Avoidability Assessment Tool. PLoS One [Internet]. 2017 Jan 3;12(1):e0169393-e0169393. Available from: https://www.ncbi.nlm.nih.gov/pubmed/28046035

10. Danjuma MI-M, Shokri S Al, Abubeker IY, Malik A El, Abdallah IMH, Shafei MN El, et al. An investigation into the avoidability of adverse drug reactions using the LAAT and modified Hallas tools. Medicine (Baltimore). 2020 Jan;99(1):e18569.

11. Danjuma MI-M, Almasri H, Alshokri S, Khir FK, Elmalik A, Battikh NG, et al. Avoidability of druginduced liver injury (DILI) in an elderly hospital cohort with cases assessed for causality by the updated RUCAM score. BMC Geriatr [Internet]. 2020;20(1):346. Available from: https://doi.org/10.1186/s12877-020-01732-3

12. Danjuma MI, Sinha U, Fatima H, Mohamed MFH. QTc evaluation in COVID-19 patients treated with chloroquine/hydroxychloroquine: A Letter to the Editor. European journal of clinical investigation. England; 2020. p. e13407.

13. Landis JR, Koch GG. The measurement of observer agreement for categorical data. Biometrics. 1977 Mar;33(1):159-74.

14. Danjuma MIM ud, Almasri H, Alshokri S, Khir FK, Elmalik A, Battikh NG, et al. Avoidability of druginduced liver injury (DILI) in an elderly hospital cohort with cases assessed for causality by the updated RUCAM score. BMC Geriatr. 2020;20(1).

15. Danjuma MI-M, Almasri H, Alshokri S, Khir FK, Elmalik A, Battikh NG, et al. Avoidability of druginduced liver injury (DILI) in an elderly hospital cohort with cases assessed for causality by the updated RUCAM score. BMC Geriatr. 2020 Sep;20(1):346.

16. Cabañas R, Calderón O, Ramírez E, Fiandor A, Caballero T, Heredia R, et al. Sensitivity and specificity of the lymphocyte transformation test in drug reaction with eosinophilia and systemic symptoms causality assessment. Clin Exp allergy J Br Soc Allergy Clin Immunol. 2018 Mar;48(3):325-33.

\section{Figures}




\section{LIVERPOOL ADVERSE DRUG REACTION AVOIDABILITY ASSESSMENT TOOL}

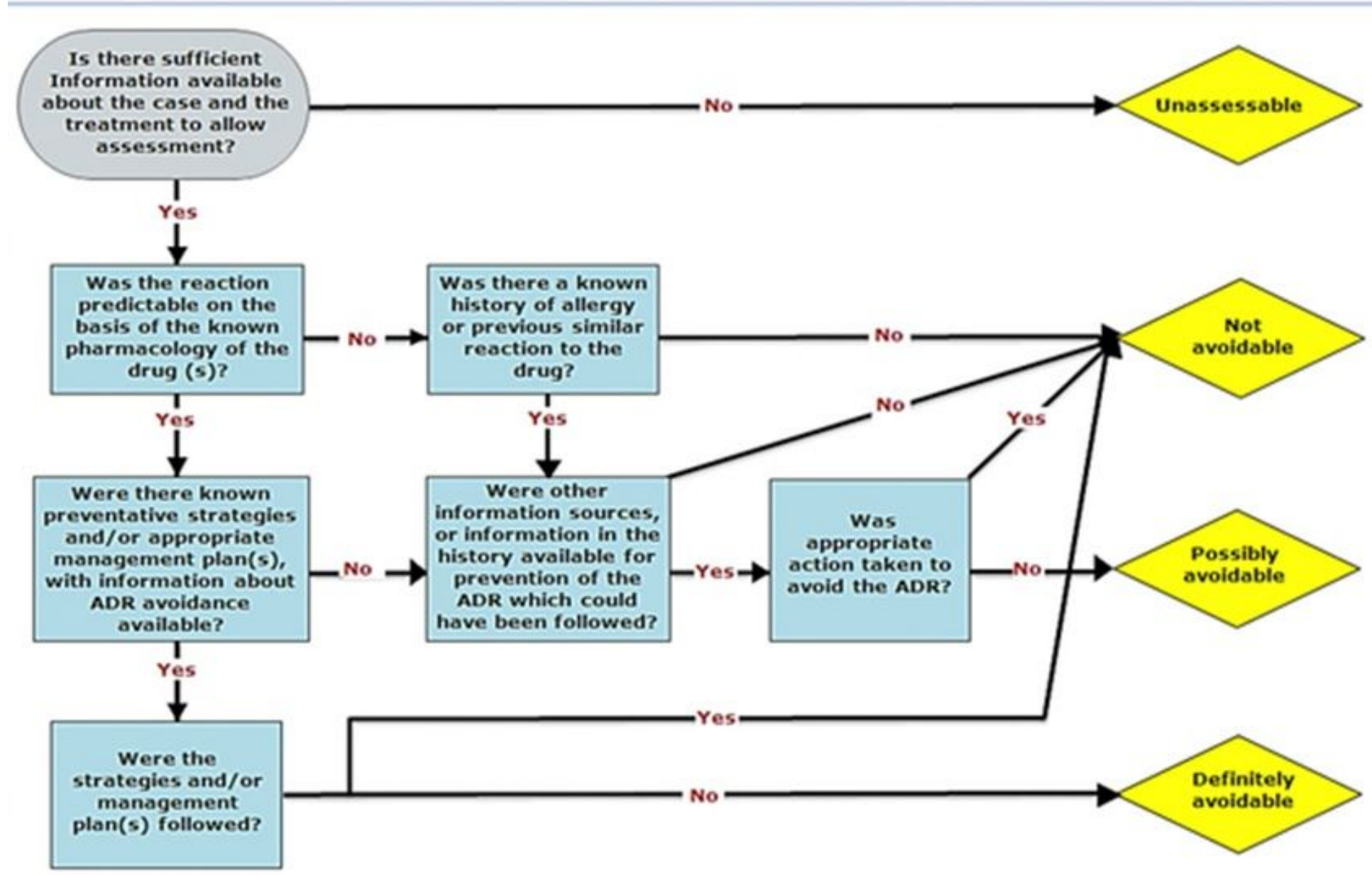

Figure 1

The Liverpool ADR avoidability assessment tool (LAAT) Adapted from [9]. 


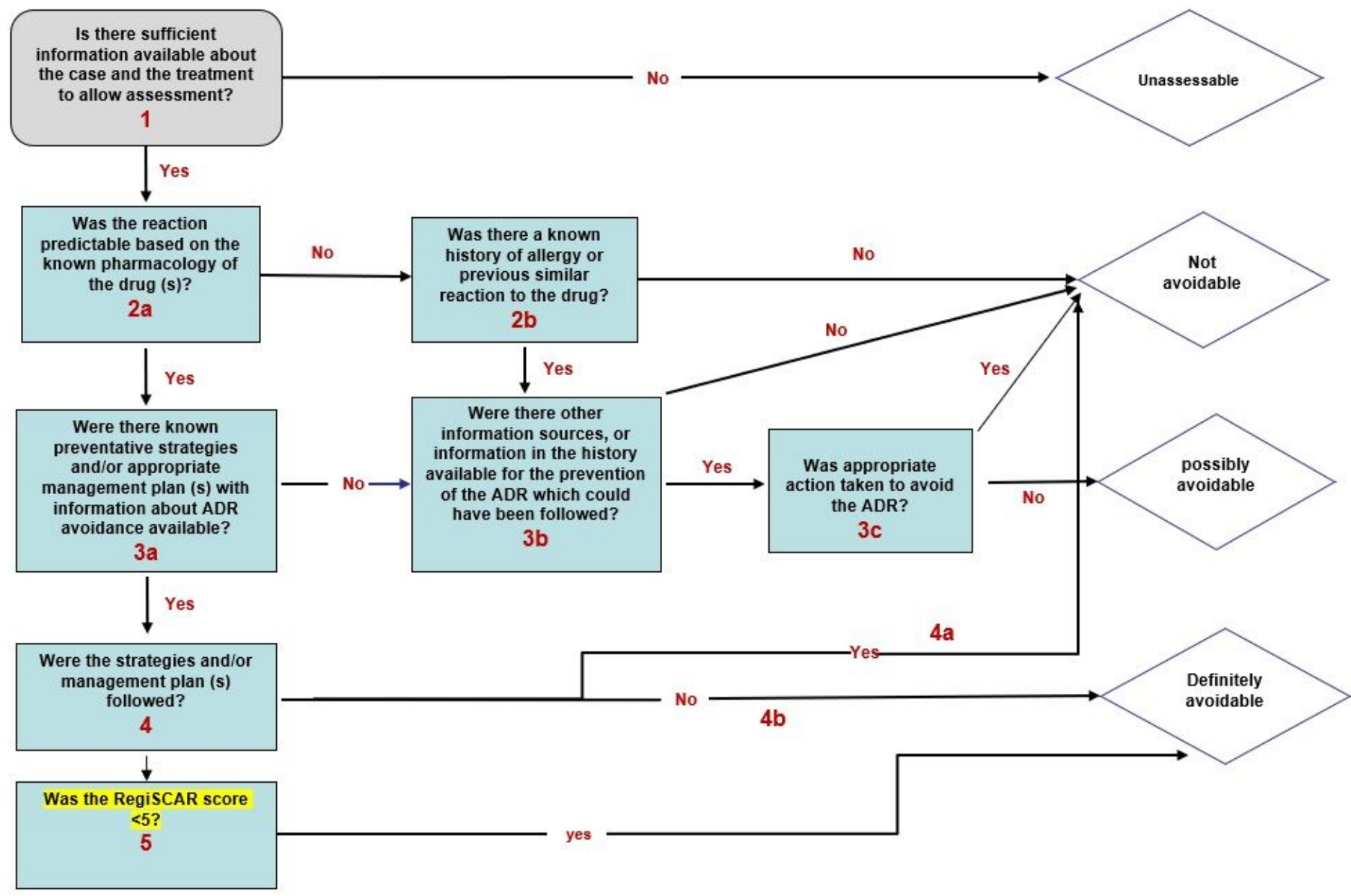

Figure 2

Schematic representation of the Liverpool adverse drug reactions avoidability tool showing the path of determination of avoidability of DRESS syndrome-Drug pairs (adapted from Bracken et al [9),

\section{Supplementary Files}

This is a list of supplementary files associated with this preprint. Click to download.

- Table1SR.docx 